\title{
Phosphorylation of silk fibroins improves the cytocompatibility of silk fibroin derived materials: A platform for the production of tuneable materials
}

\author{
Vadim Volkov ${ }^{1}$, Andreia Vasconcelos ${ }^{1}$, Marisa P. Sárria ${ }^{1,2}$, Andreia C. Gomes ${ }^{2}$, Artur Cavaco-Paulo \\ ${ }^{1}$ Centro de Engenharia Biológica, Universidade do Minho, Campus de Gualtar, Braga, Portugal \\ ${ }^{2}$ Centro de Biologia Molecular e Ambiental (CBMA), Departamento de Biologia, Universidade do Minho, Campus de Gualtar, \\ Braga, Portugal
}

Silk fibroin demonstrates great biocompatibility and is suitable for many biomedical applications, including tissue engineering and regenerative medicine. Current research focuses on manipulating the physico-chemical properties of fibroin, and examining the effect of this manipulation on firobin's biocompatibility. Regenerated silk fibroin was modified by in vitro enzymatic phosphorylation and cast into films. Films were produced by blending, at several ratios, the phosphorylated and un-phosphorylated fibroin solutions. Fourier transform infra-red spectroscopy was used to determine the specific $\mathrm{P}-\mathrm{OH}$ vibration peak, confirming the phosphorylation of the regenerated silk fibroin solution. Differential scanning calorimetry showed that phosphorylation altered the intra- and inter-molecular interactions. Further experiments demonstrated that phosphorylation can be used to tailor the hydrophylicity/hydrophobicity ratio as well as the crystalinity of silk fibroin films. Release profiling of a model drug was highly dependent on silk modification level. Cytotoxicity assays showed that exposure to lixiviates of phosphorylated films only slightly affected cellular metabolism and proliferation, although direct contact resulted in a strong direct correlation between phosphorylation level and cell proliferation. This new method for tuning silk biomaterials to obtain specific structural and biochemical features can be adapted for a wide range of applications. Phosphorylation of silk fibroins may be applied to improve the cytocompatibility of any silkbased device that is considered to be in contact with live animals or human tissues.

$\begin{array}{ll}\text { Received } & \text { 02 MAY } 2014 \\ \text { Revised } & \text { 09 JUN 2014 } \\ \text { Accepted } & 31 \text { JUL } 2014 \\ \text { Accepted } & \\ \text { article online } & \text { 03 AUG } 2014\end{array}$

Supporting information available online

Keywords: Kinases · Silk fibroin · Silk phosphorylation · Textile biotechnology

\footnotetext{
Correspondence: Prof. Artur Cavaco-Paulo, CEB - Centro de Engenharia Biológica, Universidade do Minho, 4710-057 Braga, Portugal.

E-mail: artur@deb.uminho.pt
}

Abbreviations: Ala, alanine; Arg, argine; ATP, Adenosine-5'-triphosphate; DSC, differential scanning calorimetry; FTIR, Fourier transform infra-red spectroscopy; Gly, glycine; Leu, leucine; MeOH, methanol; MTS, 3-(4,5-dimethylthiazol-2-yl)-5-(3-carboxymethoxyphenyl)-2-(4-sulfophenyl)-2Htetrazolium; PBS, phosphate-buffered saline; phospho-Ser, phosphorylated serine; Ser, serine; Tg, glass transition temperature; Thr, threonine; Tyr, tyrosine

\section{Introduction}

Silks are naturally occurring protein polymers produced by a variety of insects and spiders [1]. Silk fibroin from silkworm Bombyx mori consists of two proteins: light (25 kDa) and heavy (391 kDa) chains, produced in a 1:1 ratio and linked by a disulphide bond [2]. The amino acid composition of a heavy chain consists mostly of glycine (Gly), alanine (Ala), and serine (Ser) in the molar ratio of 3:2:1, which form typical -(-Ala-Gly- $)_{n}$ - hydrophobic motifs known as $\beta$-sheet crystalline domains [3]. Based on silk fibroin biocompatibility, biodegradability, and mechanical strength, different silk-derived materials have been developed for biomedicine [4-6]. 
Several properties of silk, such as mechanical characteristics, solubility, and biodegradability, can be controlled by manipulating silk's structure. Silk proteins have been genetically engineered in a variety of host systems [7]. Furthermore, multi-component systems of silk fibroin blended with various substances have been produced [8, 9]. Silkbased materials may be selectively degraded to modulate their porosity, and their surface may be altered by coupling peptides or other molecules of biological significance [10-12]. Amino acid residues in silk such as Tyr and Ser can be modified enzymatically $[13,14]$ a property which can be exploited for functionalizing silk-based materials.

Phosphorylation is one of the most important posttranslational modifications of proteins, and incorporation of a single phosphate group can establish many strong hydrogen bonds that affect intra- and inter-molecular interactions [15]. Protein phosphorylation is stable under physiological conditions, thus suitable for directing the formation and reorganization of dynamic protein interaction networks [16]. However, using phosphorylation for silk fibroin protein functionalization studies is largely unexplored. Only one report on silk fibroin phosphorylation in vitro has been published so far [17]. The authors described the phosphorylation of a recombinant spider dragline silk fibroin variant with the engineered kinase recognition sequence in order to increase silk fibroin solubility [17]. In turn, we were interested in modifying by phosphorylation $B$. mori reconstituted silk fibroin heavychain proteins. To the best of our knowledge, this is the first study attempting such a modification of regenerated silk fibroin. The enzyme protein kinase A can be used to achieve phosphorylation of silk protein, with Ser as the only possible phosphate acceptor $[18,19]$.

Biocompatibility is a pivotal aspect in developing new materials for applications in living systems. One of the most important aspects of biocompatibility is cytotoxicity, often measured in terms of cell viability or proliferation. Significant research has been devoted to fabrication of bio-inspired materials with different cell -differentiating and -sustaining features [20-22]. If the materials are not cytotoxic, they may or may not promote cell proliferation and, inversely, cell differentiation. By lowering the probability of cell attachment to the surface of the material, cell proliferation is not favored. One of the possible approaches to reduce cell attachment is to create a negatively charged bio-material surface, relying on the electrostatic repulsion between the partially negative charge of cell outer membrane and the bio-material.[23]

In this study, we demonstrate a straightforward methodology to produce biocompatible silk-based materials with tunable characteristics. Phosphorylated silk fibroin solutions were made to produce cast films of variable phosphorylated serine (phospho-Ser) content. The materials obtained were submitted to several tests that confirmed a dependency between the amount of phospho-Ser and their bio-chemical properties, including load- ing of a model pharmacological compound with antiinflammatory activity.

\section{Materials and methods}

\subsection{Materials}

Silk cocoons from Bombyx mori were supplied from Sezione Specializzata per la Bachicoltura (Padova, Italy). The L929 cell line was used. Kinase-GLO ${ }^{\circledR}$ assay kit (Cat.no. V6712) and CellTiter 96 ${ }^{\circledR}$ Aqueous One Solution (Cat.no. G358B) were obtained from Promega Corporation, USA. Petri dishes (Cat.no. 391-2072) were from VWR International. Tissue culture test plates were from TPP Techno Plastic Products AG, Switzerland. Whatman grade 2 filter paper (Cat.no. 1002-070) was from Whatman, USA. Glass culture cylinders (Cat.no. 070303-10) were supplied by Chromaphor Analysen-Technik GmbH, Germany. All other reagents were purchased from SigmaAldrich, Spain.

\subsection{Preparation of silk fibroin solution}

Reconstituted silk solution was obtained as described previously [24]. The concentration of silk was assessed via dry weight method in triplicate.

\subsection{Prediction of phospho-sites in silk fibroin}

To obtain an initial pool of possible sites, three programs: GPS (stand-alone software v2.1 [25]), KinasePhos [26], and PPSP [27] (both available online) were used to analyse the fibroin sequence (DDBJ/EMBL/GenBank accession no AF226688). High sensitivity was used in all programs, followed by filtering out false-positive predictions. For GPS, the threshold was reset to all and the results scoring $\geq 0.9$ were taken; for KinasePhos an HMM bit was set to 8.8 and all non-repetitive results taken; for PPSP high sensitivity of performance was used, only the results with risk-differential > 1.43 taken. Exact site positions, observed in any two of the tree resulting groups, were considered as phosphorylation-probable, resulted in 16 sites. Those sites, as well as the entire sequence, were inspected for the characteristic motif -Arg(Lys/Thr)-Arg(Lys/Ser/Thr)-XSer-X(Leu)-X(Ser)- [28]. Seven of the computationally predicted sites were discarded, and 11 newly identified sites were included.

\subsection{Preparation of phospho-silk fibroin films}

Dialyzed raw silk fibroin solution was enzymaticaly phosphorylated using protein kinase A (EC 2.7.1.37) at the concentration of 1 protein kinase A Unit for a $95 \mu \mathrm{L}$ reaction volume of appropriate buffer (50 mM TRIS-HCl, $10 \mathrm{mM}$ $\mathrm{MgCl}_{2}, \mathrm{pH} 7.5$ [17]). 0.5 mM Adenosine-5'-triphosphate 
(ATP) was a source of phosphate. $3 \mu \mathrm{M} \mathrm{Na}_{3} \mathrm{VO}_{4}$, a phosphatase inhibitor and $55 \mu \mathrm{M}$ 3', $5^{\prime}$-cyclic-AMP were added to the reaction mixture, followed by incubation at $20^{\circ} \mathrm{C}$ with stirring at 30-40 rpm for 5 to 6 hours $[18,19]$. The reaction progress was qualitatively assessed hourly using Kinase-GLO ${ }^{\circledR}$ kit. After the phosphorylation reaction the desired blends, containing various amounts of phosphosilk fibroin content, were prepared by casting and mixing the appropriate quantities of raw silk fibroin and phosphosilk fibroin solutions in Petri dishes. Cast solutions of $5 \mathrm{~mL}$ volume were left for drying under constant air flow in a laminar flow hood for 2 to 3 days at room temperature.

\subsection{Quantitative determination of phosphate incorporated in phospho-silk fibroin}

An aliquot of reaction mixture was initially precipitated with cold ethanol (1:9 volume ratio), centrifuged at 5000 RPM, re-suspended in double distilled water with vortexing, and incubated in a warm bath $\left(35^{\circ} \mathrm{C}\right)$ with $2.5 \mathrm{mM} \mathrm{NaOH}$ [29] for $15 \mathrm{~min}$. A 40 min centrifugation at 9000 RPM followed, and the supernatant was removed carefully. A mixture of 22 volumes of supernatant to 1 volume of $70 \% \mathrm{w} / \mathrm{v} \mathrm{HClO}_{4}$ was centrifuged at $9000 \mathrm{rpm}$ for $30 \mathrm{~min}$ [30]. The resulting supernatant was diluted with double distilled water and used for malachite green reaction ((malachite green reagent):(sample) volume ratio 1:6 $[31,32])$. The reaction mixture was incubated at $20^{\circ} \mathrm{C}$ for $30 \mathrm{~min}$ and the optical absorbance read at $630 \mathrm{~nm}$ against a standard curve.

Knowing the amount of phosphorylation sites present (Section 2.3), and the fact that each discrete site may be modified only once, the degree of phosphorylation was determined as follows:

$\frac{\text { Pi amount released }}{\text { Maximum possible Pi amount to be released }} \times 100$ (Eq. 1)

Where $\mathbf{P i}$ is the concentration of inorganic phosphate.

\subsection{Fourier transform infra-red (FTIR) spectroscopy}

FTIR spectra of different Silk fibroin films were measured with a JASCO FT/IR-4100 type A spectrometer in the spectral region of 4000-600 $\mathrm{cm}^{-1}$ with a ZnSe cell in ATR mode and $8 \mathrm{~cm}^{-1}$ resolution. The analysis was performed in 64-pass rescan mode, on two different film areas.

\subsection{Secondary structure analysis}

FTIR-derived convoluted curves, corresponding to Amide I spectral intervals of $[1600 ; 1700] \mathrm{cm}^{-1}$, were processed using the Feat Peaks (Pro) routine of Peak Analyzer in OriginPro, v.8.5.0 (OriginLab Corporation, USA). For each compound curve, the baseline subtraction was followed by a multiple pass fit. Discrete initial peak values used for fitting were identified by a Second Derivative inbuilt method. The secondary conformational data, resulting from individual peaks, were obtained basing on the reported peak assignments [33].

\subsection{Thermal analysis}

Differential scanning calorimetry (DSC) measurements were performed, in duplicate, with a DSC-30 instrument (MettlerToledo), from room temperature to $120^{\circ} \mathrm{C}$, at a heating rate of $10^{\circ} \mathrm{C} \mathrm{min}{ }^{-1}$, and kept at $120^{\circ} \mathrm{C}$ for $10 \mathrm{~min}$, to induce sample dehydration. The temperature was lowered to $25^{\circ} \mathrm{C}$ and increased to $350^{\circ} \mathrm{C}$ at a heating rate of

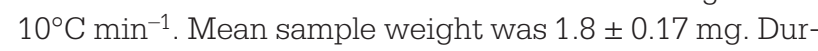
ing the analysis the aluminum cell was swept with $80 \mathrm{~mL}$ $\mathrm{min}^{-1} \mathrm{~N}_{2}$ flow.

\subsection{Swelling ratio}

Phospho-silk fibroin materials, treated with methanol $(\mathrm{MeOH})$ and completely dry $\left(30^{\circ} \mathrm{C}\right.$ for $\left.24 \mathrm{~h}\right)$ were immersed in phosphate-buffered saline (PBS 0.1 M; pH 3, 7.4 and 11) at $37^{\circ} \mathrm{C}$ for $24 \mathrm{~h}$. The excess buffer was removed and the material wet weight was determined. The swelling ratio was calculated as follows:

$$
\frac{\mathrm{Ws}-\mathrm{Wd}}{\mathrm{Wd}}
$$

Where Ws is the mass of the swollen material and $\mathbf{W d}$ is the initial dry mass. This analysis was performed in duplicate.

\subsection{Time of adsorption of water drop}

Using a micropipette a droplet of water was left over the surface of films. Time was counted until liquid was fully spread. Duplicates were performed.

\subsection{In vitro release of loaded piroxicam from the elaborated films}

The release of $1.8 \mathrm{mM}$ incorporated piroxicam was studied. Piroxicam was initially dissolved in dimethyl sulfoxide and an equal volume of double distilled water. Control phosphosilk samples were cast devoid of piroxicam and all the materials were treated with $\mathrm{MeOH}$. $\mathrm{MeOH}$ solution was collected from each sample and the released piroxicam due to alcohol application was quantified. Phospho-silk fibroin films were incubated at room temperature under constant shaking (50-60 rpm) in PBS (0.1 M, pH 7.4) or in a solution containing protease, subtilisin from Bacillus sp. (EC 3.4.21.62), at $0.075 \mathrm{U} \mathrm{mL}^{-1}$ [34]. Piroxicam release was quantified by absorbance measurements at $353 \mathrm{~nm}$ against standard curves. No buffer exchange was performed throughout the entire assay. All the release experiments were carried out in duplicates for 5 days. The release 
behavior was determined by fitting the experimental data to the empirical relationships given by Ritger-Peppas (3) and Higuchi simplified (4) equations [35, 36].

$\frac{\mathrm{M}_{\mathrm{t}}}{\mathrm{M}_{\infty}}=\mathrm{kt}^{\mathrm{n}}$

$\mathrm{f}_{\mathrm{t}}=\mathrm{K}_{\mathrm{H}} \sqrt{\mathrm{t}}$

Where $\frac{M_{t}}{M_{\infty}}$ is a released piroxicam fraction, calculated as released at discrete incubation time $t$

ratio;

what is left to be released after $\mathrm{MeOH}$ treatment ratio;
$\mathbf{k}$ is a constant incorporating structural and geometric characteristics of the drug dosage form; $\mathbf{n}$ is the release exponent and obtained by a linear fitting of log(fraction) versus log $t$ from the modified equation (3):

$\log ($ fraction $)=\log \mathrm{k}+\mathrm{n} * \log \mathrm{t}$

$\mathbf{f}_{\boldsymbol{t}}$ is the amount of drug released at discrete incubation time and $\mathbf{K}_{\mathbf{H}}$ is the Higuchi dissolution constant. According to the model, $\mathbf{K}_{\mathbf{H}}$ is obtained from a linear fitting of $\mathrm{f}_{t}$ as a function of square root of time $(\mathrm{t})$, where $\mathbf{K}_{\mathbf{H}}$ is a line slope. The results obtained are reported as averages with standard deviations.

\subsection{L929 fibroblast-like mouse cell culture}

The L929 fibroblast-like mouse cell line (ATCC ${ }^{\circledR}$ CCL1 ${ }^{\mathrm{TM}}$ ) was maintained as described previously [37]. For experiments, cells were seeded at a concentration of $5.5 \times 10^{4}$ cells $\mathrm{mL}^{-1}$.

\subsection{Proliferation assays of L929 fibroblast-like mouse cells}

3-(4,5-dimethylthiazol-2-yl)-5-(3-carboxymethoxyphenyl)2-(4-sulfophenyl)-2H-tetrazolium (MTS), in the presence of phenazine ethosulfate, is bio-reduced by cells into a formazan product with an absorbance maximum at $490 \mathrm{~nm}$, assaying active cell metabolism [38]. Sulforhodamine B is a pink aminoxanthine dye of absorbance maximum at $540 \mathrm{~nm}$, adsorbed by basic amino acids in the cells in proportion to cell number and indicative of cell proliferation [39]. Triplicate replicates for each individual assay were done.

\subsubsection{Biocompatibility test of elaborated materials by indirect contact}

Phospho-silk films were disinfected by triple washings with antibiotic/antimycotic solution, placed into 12-well culture plates (each film in a separate well) and culture medium (without fetal bovine serum) was added. After an incubation of 1 or 3.5 days at $37^{\circ} \mathrm{C}$, the medium was harvested and supplemented with fetal bovine serum. Addi- tionally, $0.6 \mathrm{~g} \mathrm{~L}^{-1}$ of L-glutamine was added to 3.5-days conditioning batch by the end of conditioning period. The conditioned medium was then applied to adhered L929 cells, replacing the regular medium. The cells were incubated for 48 and 72 hours and the proliferation was assessed by MTS or sulforhodamine B techniques.

\subsubsection{Biocompatibility test of elaborated materials by direct contact}

After disinfection, $10 \mathrm{~mm}$ circles were excised from each hydrated film type using a borer and placed in 24-well culture plate, ensuring a flat contact area between the surfaces. Glass culture cylinders were placed on top of the film and $250 \mu \mathrm{L}$ of cell suspension solution was applied to the inner area of each cylinder. After 24 hours of contact the cells were stained with $1 \mu \mathrm{g} \mathrm{mL} \mathrm{m}^{-1}$ Hoechst 33258 for 15 minutes. Cellular adhesion was visualized by epifluorescent bright field microscopy (Olympus IX71 controlled by $\mathrm{Cell}^{\wedge} \mathrm{F}$ Olympus Imaging Solutions software v3.4, $\mathrm{GmbH}$ ). The quantity of cells was assessed using the Analyze Particles routine of ImageJ v1.42h using the fluorescence emitted from Hoechst 33258-stained nuclei. Three different regions were examined of each film type.

\subsection{Statistical analysis}

In order to detect differences between phosphorylation treatments in the swelling ratio, a $t$-test was conducted with the observed values. A piecewise regression was conducted in order to detect the breaking point of the swelling ratio curve. Percentage of phospho-content was used as the dependent variable (least square method was applied). The detection of the breaking point had two separate purposes: (i) to evaluate if different $\mathrm{pH}$ conditions showed different breaking points; and (ii) to determine the percentage of phospho-content that corresponds to the inflection value.

Prior to analysis all cell proliferation-related data were tested for normality (Shapiro-Wilk test) and homogeneity of variances (Levene's test). To investigate the influence of the modification degree on cell proliferation, a threelevel nested design ANOVA test was conducted comparing the type of bio-film matrix, conditioning period, and timepoint. Post hoc comparisons were conducted using Student-Newman-Keuls. A P value of 0.05 was used for significance testing. Analysis were performed in STATISTICA (StatSoft v.7).

\section{Results}

\subsection{Chemical aspects of preparation of phospho-silk fibroin films}

Our work used regenerated mulberry silk, which required the establishment of a suitable protocol for its phosphory- 


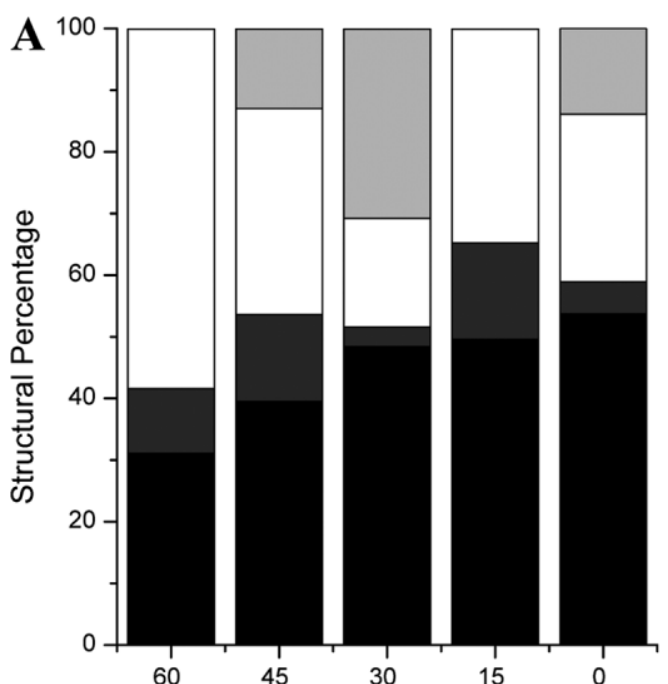

Film Type

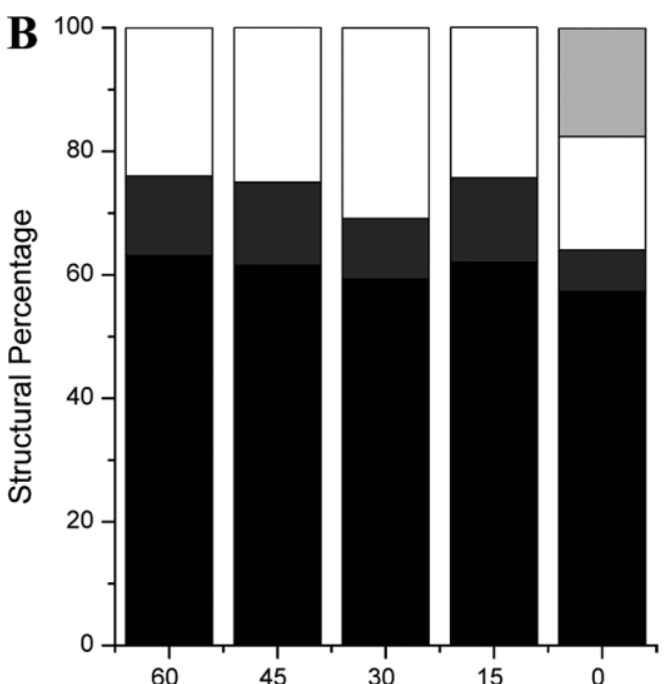

Film Type

3[10] Helix $\square$ Random

Beta-related

Beta-sheet

Figure 1. Dependency between phosphorylated content and the observed silk fibroin protein structure. Amide I resolved curve data for different Silk Fibroin film secondary structures. (A) Dried films with no treatment. (B) Dried films, MeOH-treated. $3[10] \mathrm{Helix}=3_{10} \mathrm{Helix}$; Random $=$ Random coiled structure; Beta-related $=\beta$-related turns. The data are based on one experiment with double sampling.

lation. Previously, Winkler performed the phosphorylation of recombinant dragline silk using protein kinase A [17, 19]. Different protein kinase A concentrations, ATP and 3',5'-cyclic-AMP concentrations, and raw silk fibroin solution quantities were tested. Supporting information, Fig. S1 demonstrates that a 6:1 volume ratio between silk solution and reaction buffer (constituting $\approx 161 \mathrm{mg}$ of silk fibroin in $10 \mathrm{~mL}$ reaction volume) is the most favorable for the phosphorylation reaction.

To calculate the exact level of phosphorylation, quantification of phosphate ion (Pi) was performed by the malachite green method [40]. Pi originated from dephosphorylation of silk by $\beta$-elimination (see Section 2.5). Malachite green indicates that phosphorylation can reach $\approx 60 \%$ of all predicted sites. Similar values have been reported previously [17]. Of all amino acids constituting the fibroin chain, and that could undergo phosphorylation (Ser, Tyr, Thr), only Ser is a phosphate acceptor as Tyr is not a substrate for protein kinase A [18] and Thr is $\approx 0.89 \%$ of the amino acids present. It was estimated that a small portion of Ser (19 residues, or 3\% of its total amount) and the single Thr can be phosphorylated (see [3] and Supporting information, Fig. S2).

The phospho-silk fibroin solution was prepared and diluted to the desired phosphorylation level, obtaining the four solutions (designated as $60 \%, 45 \%, 30 \%$ and $15 \%$ ). Those solutions were cast to form films. The dilutions were made with unprocessed silk fibroin solution which was also casted separately and served as a negative control (0\%). The phospho-content in five distinct batches of prepared films was evaluated and is presented in Supporting information, Table S1.

Methanol (MeOH) treatment, an indispensable step of the workflow, was made on a separate group of prepared materials. The $\mathrm{MeOH}$ treated group, and the untreated one, were further examined and compared in parallel, as described throughout this article.

\subsection{Chemical and physical properties of modified silk fibroin}

\subsubsection{FTIR analysis reveals the structural dependence on phosphorylation}

FTIR analysis showed that, regardless of $\mathrm{MeOH}$ treatment, characteristic absorbance spectra could be obtained from the two groups (Supporting information, Fig. S3). The fibroin conformation, reported for the untreated films is predominantly random coil and corresponds to Amides I-II [41, 42]. Such random coils suggest the prevailance of an amorphous structure, mainly consisting of $\beta_{\mathrm{I}}$-turns and anti-parallel chain-pleated sheets (Amide I). $3_{10}-$ and $\alpha$ - helixes [43] are also encountered (Amide II). The proximity of IR frequencies of both helical structures makes it difficult to distinguish between them [44]. Yet, the results of our structural analysis indicate that $\beta$-related structures ( $\beta$-sheets and $\beta$-turns) can constitute at least $40 \%$ of the secondary silk fibroin conformation even in the untreated group (Fig. 1A).

Secondary structure conformations of silk were found to be phosphorylation-dependent. The incorporation of 
phosphate disrupts the $\beta$-sheet organization of fibroins [17]. These results demonstrate that the films with lower phosphate content have enhanced $\beta$-sheet formation, in line with previous studies $[41,42,45]$. Upon alcohol application, the total amount of $\beta$-structures increased to $\approx 72 \pm 5.3 \%$ (specifically $\approx 60.6 \pm 2.3 \%$ of $\beta$-sheets) of the total protein conformation (Fig. 1B). The formation of $\beta$-related turns, associated with $\beta$-sheet stacking, also increased [46].

\subsubsection{DSC analysis confirms plasticizing effect of phospho-groups on fibroin-derived films}

The interactions within phospho-fibroin films were further investigated by DSC. The corresponding DSC scans are shown in Fig. 2A and $2 \mathrm{~B}$, and the main thermal peaks identified are presented in Supporting information, Table $\mathrm{S} 2$. A characteristic thermal trend was obtained for $\mathrm{MeOH}$ treated and untreated samples. Upon temperature rising, residual water evaporation, glass transition, crystallization and the decomposition of material take place [24, 47]. After phosphorylation the intermolecular hydrophobic interactions within silk fibers decrease [48]. Thermodynamic parameters such as heat capacity depend on these interactions [49]. A complex tendency can be demonstrated in the untreated group, which clearly defines two types of curves - related to more (0 and 15\%) and less (30, 45 and 60\%) aggregated materials. The order in which the $\mathrm{MeOH}$-untreated materials absorb energy (in terms of measured heat flow) is different from the order in which the untreated materials adsorb energy.

The glass transition temperature ( $\mathrm{Tg}$ ) is inversely dependent on intermolecular interactions and protein flexibility [48]. Tg and the crystallization peaks deter- mined herein were found to be dependent on phosphoSer content (Supporting information, Table S2). A higher percentage of phosphorylation results in lower $\mathrm{Tg}$ and crystallization onset values. Yet, $\mathrm{Tg}$ and crystallization peaks could not be determined for the $\mathrm{MeOH}$-treated materials. The transition from random coil to $\beta$-sheet is observed after $\mathrm{Tg}$ in untreated films and if $\beta$-sheet was induced prior to DSC analysis, Tg and crystallization peaks became unrecognizable. [47, 50, 51]. The $\mathrm{MeOH}-$ treated group heat flow curves preserve their spectral localization with temperature increases. The higher uniformity of the treated group is dictated by alcoholinduced dehydration and partial pre-crystallization that counteracts the effects introduced by phosphate groups. The treated group exhibits a much more pronounced decomposition of endotherm peaks, indicating that the fibroin proteins building the films are found in a more crystalline $\beta$-sheet state.

\subsection{In vitro swelling of $\mathrm{MeOH}$-treated films}

Swelling behavior of different $\mathrm{MeOH}$-treated films was examined. Non MeOH-treated materials could not be examined since they degrade rapidly in aqueous media. The experimental outcome is presented in Supporting information, Fig. S4. From the acquired data, three important conclusions can be drawn: i) the material swelling is phosphorylation-dependent and is highest when 30\% of the starting silk fibroin is phosphorylated; ii) the swelling is $\mathrm{pH}$-dependent and is most pronounced at basic $\mathrm{pH}$ value; iii) a moderate increase in the tendency to swell exists at a phosphorylation range between $0 \%$ and $30 \%$.
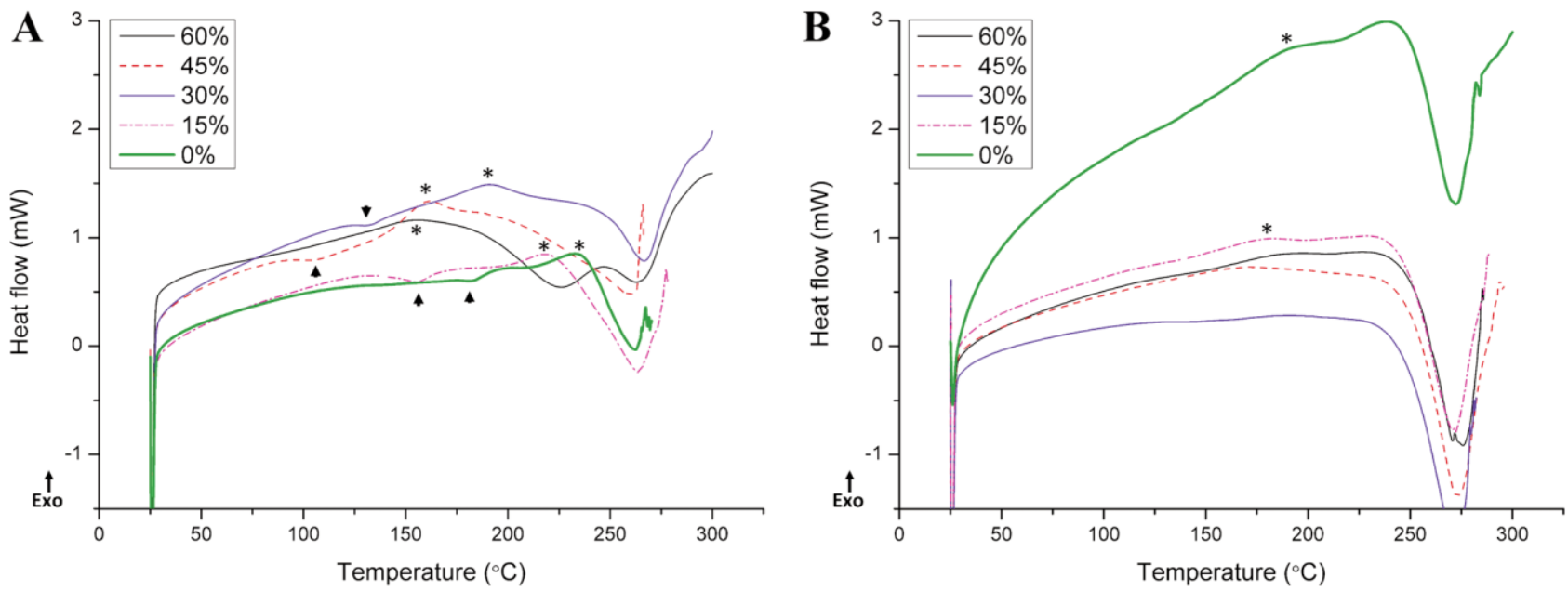

Figure 2. Differential scanning calorimetric analysis of (A) untreated, (B) MeOH-treated blended phospho-fibroin films. Individual thermograms of the corresponding samples are shown. $\mathrm{Tg}$ values are shown for the untreated group (marked by the $\downarrow$ symbol). Crystallization endotherms are designated by the * symbol. Converse to the $\mathrm{MeOH}$-treated samples (B), a shift towards lower glass transition temperature and materials' grouping (condensed 0\%, 15\%; light $30-60 \%$ ), as function of phosphorylated content, are recognized for untreated phospho-silk fibroin films (A). The data are based on one experiment with double sampling. 
When analyzing the results of the $t$-test's, the swelling ratio values were shown to be significantly different considering the percentage of P-Ser content for all $\mathrm{pH}$ conditions tested ( $t$-value $(4)=2.7 ; P<0.05$ ). Interestingly, independent of the $\mathrm{pH}$ condition, the estimated breakpoint occurred, in all treatments, invariably at 30\% of phosphorylation. Further discussion on this phenomenon is found in Section 4.

\subsection{In vitro release of piroxicam from MeOH-treated films}

For release evaluation piroxicam was chosen as a model compound. Piroxicam is a non-steroidal anti-inflammatory drug, used to relieve inflammatory-derived symptoms, such as those common to rheumatoid and osteoarthritis, and postoperative pain, with good efficacy. Being a relatively small and non-polar molecule, piroxicam is particularly adequate for elucidation of alterations in hydrophobicity of fibrous material. To mimic the internal body environment, PBS-buffered protease or PBS buffered isotonic solutions were used. The release profiles reveal that the drug dissipates from films bi-phasically (Fig. 3A and 3B). The initial burst phase reflects the loss of piroxicam molecules possibly located very close to the film surface and occurs during the first hour. This burst is followed by slower release, decreasing over the remaining time. Prolonged incubation ( $>2.5$ days) may cause subtle drug degradation, [52] and could result in decline of release profiles. Piroxicam release is marked when fibroin films were incubated with protease except those with 15\% phosphorylation. This effect is leveled out for highly phosphorylated films (30, 45 and 60\%). It is worth noticing that the graphs in Fig. 3 also depict the MeOH-related release prior to incubation (-1 hour time point). A significant amount of piroxicam is released during insolubility induction (starting from $\approx 11.3 \%$ of total drug amount for $0 \%$ phospho-fibroin up to $55.5 \%$ of the total drug amount for $60 \%$ phospho-fibroin). A 5-day incubation resulted in $33.5 \pm 8 \%$ and $28 \pm 2 \%$ additional release of piroxicam for subtilisin- and PBS-mediated incubation, respectively. These values are derived from averaging release profiles of fibroin films in each incubation group. No complete release occurred in the timeframe of the experiment.

Various models have been proposed for handling the release profile data, though for the case of thin slabs or solid matrixes two are commonly used: Ritger-Peppas semiempirical model and Higuchi model [35, 36, 53]. We exploited the above models to calculate the parameters of interest: constant $\mathbf{k}$; diffusion or release exponent, $\mathbf{n}$; and Higuchi dissolution constant, $\mathbf{K}_{\mathbf{H}}$. The parameters are listed in Supporting information, Table S3, designated as Sample, except the 0\% immersed in PBS sample, since it did not result in any considerable release. Time interval $\mathbf{t}$, an additional variable used in the calculations of the models, is presented in Supporting information, Table S3. t values have a unit of hours and differ from each other, since they depend on the release dynamics and on the extra conditions that each model may pose. Some of the time intervals used for the Ritger - Peppas modeling are shorter than those, used in the Higuchi model. The Ritger - Peppas equation (3) imposes that only the profile data, corresponding to the initial $60 \%$ of the released compound, may be included in kinetic calculations [35, 53]. The Higuchi model does not have such a limitation, thus the full range of burst release values can be used [53]. The
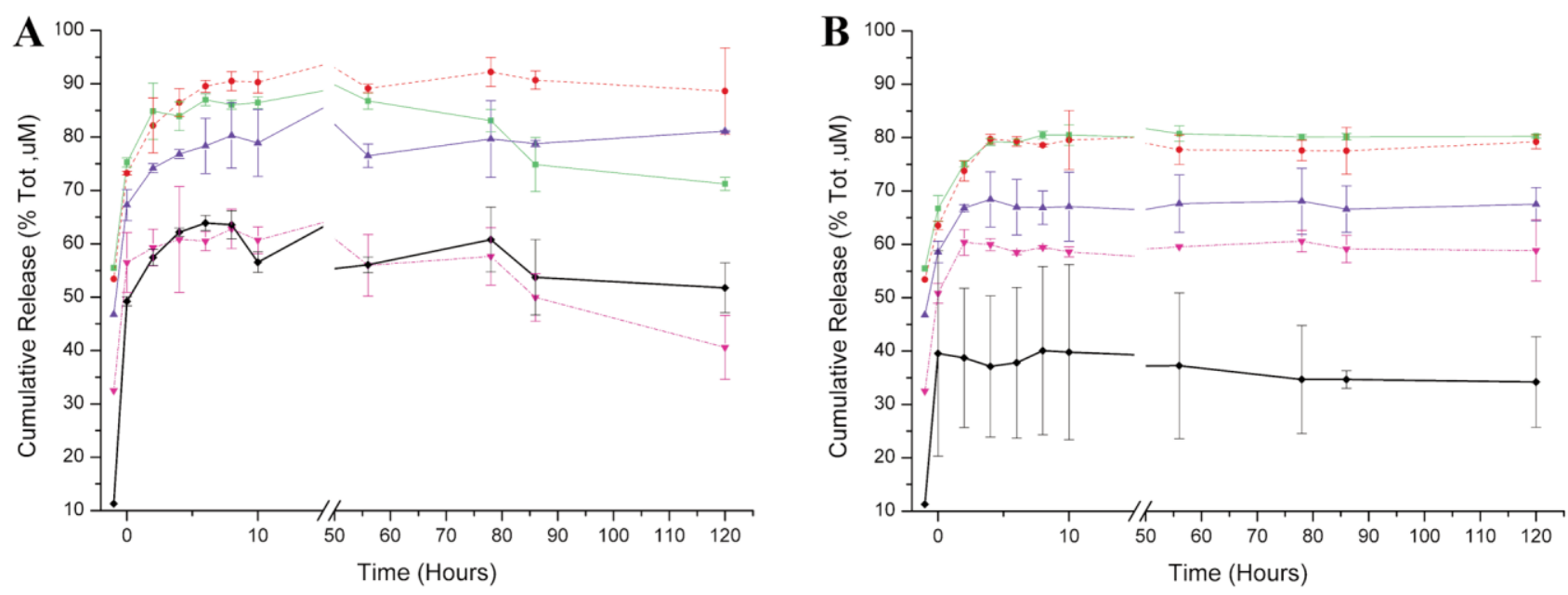

$$
=60 \% \quad-45 \% \multimap 30 \% \quad-15 \% \multimap-0 \%
$$

Figure 3. Piroxicam release profiles from silk fibroin films: (A) enzymatically (subtilisin) mediated release. (B) non-enzymatically (PBS buffer) mediated release. Note the arbitrary -1 hour time point, corresponding to $\mathrm{MeOH}$ treatment-mediated release of the drug. Cumulative release encompasses the total release of the drug, resulting from $\mathrm{MeOH}$ pre-treatment and incubation media as well. The graph is based on one experiment with double sampling. 
output of the Ritger-Peppas model provides the researcher with indication of the release mechanism and characteristics of the macromolecular network system and the drug, deduced from $\mathbf{n}$ and $\mathbf{k}$ values, respectively. The calculated $\mathbf{n}$ values indicate a non-Fickian release mechanism (anomalous diffusion), as they are below the value of 0.5 . $\mathbf{k}$ can sometimes be seen as significant interaction between the drug and its trapping material [54]. The two kinetic models can be compared through the $\mathbf{k}$ and $\mathbf{K}_{\mathbf{H}}$ values. Since neither $\mathbf{k}$ nor $\mathbf{K}_{\mathbf{H}}$ have an unambiguous definition (although describing similar concepts), it can be examined whether they originate from the same distribution or not [53]. That is, the degree of similarity among them can be assessed. A Mann-Whitney $U$ test was performed on these two sets of values, indicating a similarity between the sets [55]. The twotailed $p$-value of 0.34 that was obtained is above the defined threshold of 0.05 , therefore it cannot be out ruled that the numerical sets obtained with the Ritger-Peppas and Higuchi models are similar. Thus in this case the two modeling approaches can be used to analyze the data.

\subsection{Phosphorylation of silk fibroin improves cyto- compatibility of silk fibroin derived materials}

To validate silk fibroin films for contact with cells and tissues, cellular proliferation as a result of indirect contact (by exposure to media pre-incubated with the films), and direct contact (cells seeded on the films) were examined. The outcomes of these experiments are presented in Fig. 4 and 5. For indirect contact, the results show a significant inverse correlation between phosphorylation percentage and cell proliferation (MTS-estimated, $P<0.05$; sulforhodamine B-estimated, $P<0.05)$. Nevertheless, cell viability (i.e. metabolism as assayed by MTS) is only meaningfully affected $(<60 \%)$ by lixiviates from films with 60 or $45 \%$ phosphorylation (Fig. 4).

For a comparison between different film modifications, direct contact of cells with phosphorylated films was quantatively monitored by counting the number of adhered cells. Cell counts are presented in Supporting information, Table S4 and a representative image is depicted in Supporting information, Fig. S5. The results
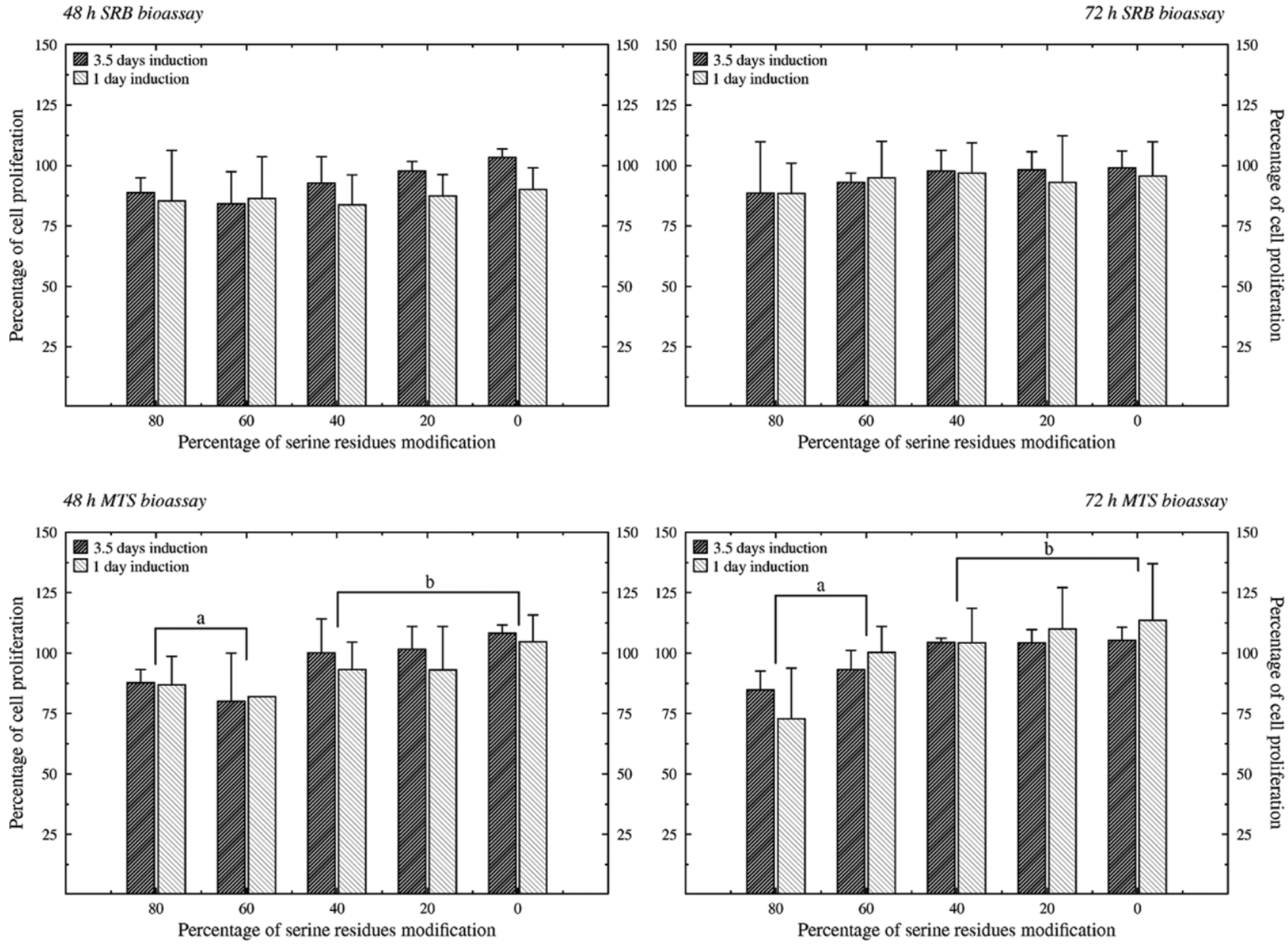

Figure 4. The effect of modification degree on cell proliferation, in direct contact measured by MTS and sulforhodamine B assays. Different letters (a, b) indicate significant differences among treatments. The resulting data are based on three independent experiments. 


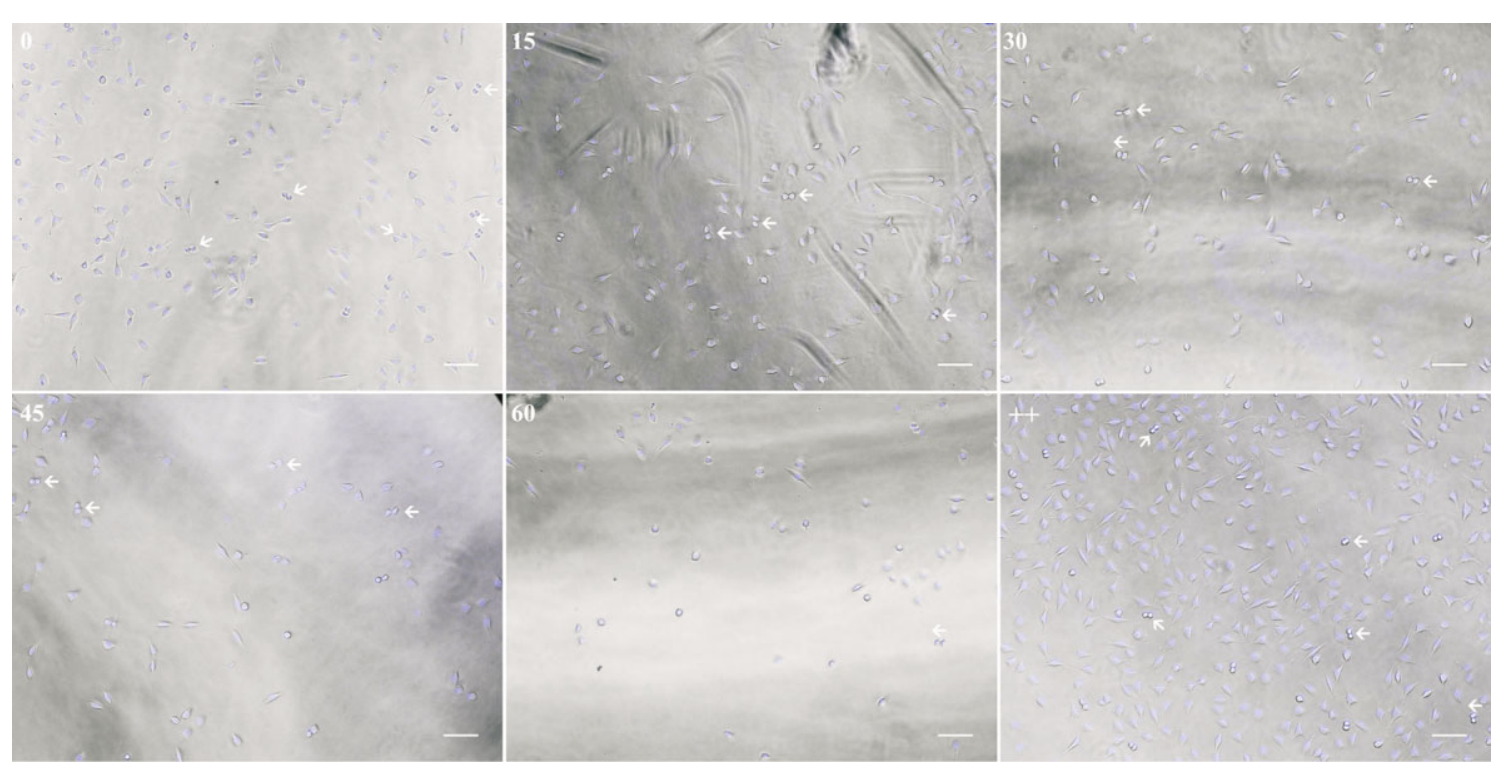

Figure 5. Fluorescence microscopy of cellular proliferation on silk fibroin films with different degrees of phosphorylation. ++ designates a positive control, the nuclear stain is Hoechst. Some of the newly divided cells are identified with arrows. Scale bar, $50 \mu \mathrm{m}$. Each film of distinct phosphorylation level was filmed twice, one of the representative images is shown.

demonstrate an inverse correlation between cell proliferation and film modification degree. The inferior cell proliferation rate per se does not necessarily signify cell death, as the environment may disfavor proliferation, while being non-toxic $[56,57]$. The latter idea is concordant with the water droplet spreading times, measured for the produced films (Table 1). A decrease in hydrophobicity correlates with more phosphate present in the film structure. Cell attachment is known to be impeded by increasing hydrophilicity $[58,59]$.

\section{Discussion}

Winkler and co-workers established a method to control the secondary structure of a genetically engineered fragment of spider dragline silk (of $\approx 25 \mathrm{kDa}$ ) by means of phosphorylation [17]. The protein used in their study was purified from a bacterial expression host. We were interested in modifying regenerated mulberry silk fibroin, bearing in mind the expected impact of phosphorylation. Prior to the enzymatic reaction, the number and location of possibly modified sites in the fibrous protein were determined in silico. Information of primary importance was the number of sites, since it made it possible to calculate the phosphorylation level. The exact positions of modification were not known. If one considers a maximum of 12 phosphorylations for each fibroin molecule (60\% of 20 sites), the minimal amount of phosphorylations intended to affect $\beta$-structures formation will constitute only half. Six other modifications, then, are located on the $\mathrm{N}$ - and $\mathrm{C}$ - terminal hydrophilic domains, therefore not contributing to $\beta$-sheet disruption (Supporting information, Fig. S2). Yet, the data of DSC and release profiling strongly support the dependency of fibroin structure on phosphate content. This means one of the following: (i) the majority of phosphorylation occur within the hydrophobic core; or (ii) the consequences of structural alteration are evident already at $\approx 43 \%$ phosphorylation (6 of 14 inner sites, located in the hydrophobic part of the protein). Similar to Winkler's

Table 1. Water droplet spreading time as a function of percent phosphorylation of fibroins ${ }^{\text {a) }}$

\begin{tabular}{ccccc}
\hline Silk fibroin blend type & Adsorption, Untreated ${ }^{b}$ [ $[\mathrm{min}]$ & Standard deviation & Adsorption, Treated ${ }^{\mathrm{c}}$ [min] & Standard deviation \\
\hline $60 \%$ & 5.23 & 0.806 & 11.83 & 1.173 \\
$45 \%$ & 8.15 & 1.202 & 14.65 & 0.919 \\
$30 \%$ & 9.25 & 0.353 & 16.75 & 1.060 \\
$15 \%$ & 13.9 & 1.272 & 19.35 & 1.626 \\
$0 \%$ & 20.25 & 1.767 & 25.35 & 0.919 \\
\hline
\end{tabular}

a) The table is based on the results of two independent assays.

b) Untreated, are materials not treated with $\mathrm{MeOH}$.

c) Treated, are materials treated with $\mathrm{MeOH}$. 
report, we observed that phosphorylation of silk fibroin caused a decrease in the hydrophobic content of the protein, but did not completely inhibit $\beta$-sheet assembly.

We observed an opposing order of energy absorption as a function of phospho-content between the two treatment groups, as visualized by DSC. This may result from two stabilizing effects: (i) innate crystallization or hydrophobic self-association of silk fibroin; and (ii) polar or dipole-induced protein stabilization introduced by the phosphate group. Proteins with a highly negative value of net charge per residue tend to reduce the number of negative-negative contacts in order to accommodate the phosphate group [60]. For proteins with a moderate net charge per residue, an increase of negative-negative contacts might correlate with stronger structural stabilization and reduced hydrophobicity [60]. The local decrease of hydrophobicity near phospho-sites is approximately constant. Such effects in the case of silk fibroin strongly interferes with hydrophobic attractions between Ala and Gly. A similar stabilization effect was previously reported for other proteins as well as the formation of stable pairs between Arg and phospho-Ser residues [61, 62].

Phosphorylation influences swelling in a way that is consistent with materials with no, or a reduced level of, modification being is less solvent accessible while extensively phosphorylated material ( $\geq 45 \%$ ) has a too relaxed structure. In the first case, it may be difficult for the solvent to penetrate the hydrophobic domains. On the contrary, at high phosphorylation solvent enters freely but is not captured within the material and therefore does not contributing to swelling. Accordingly, piecewise linear regression with breakpoints underlined the fact that elevated phosphorylation correlates with increased swelling. However, this tendency only holds in the range $0 \%-30 \%$ of modification. More pronounced phospho-content results in decreased swelling. Since the phospho-group of Ser has a pkA 1 of 2.19 and $\mathrm{pkA}_{2}$ of 5.78 [63], the double negative charge will be present on modified Ser at neutral and basic $\mathrm{pH}$. This fact explains why the swelling ratio of films is greater at basic $\mathrm{pH}$, but not acidic $\mathrm{pH}$. Moreover, below $\mathrm{pH} 5$ there is protein clustering due to the dominance of inter-molecular hydrogen bonding, which correlates with the theoretical isoelectric point of silk fibroin (pI = 4.53) [64]. Extreme pH values may induce molecular contraction within the silk fibroin. It was demonstrated that acidic $\mathrm{pH}$ promotes silk insolubility in aqueous media [64]. The electrostatic free energy of a protein is $\mathrm{pH}$ dependent and influences its stability and conformation. This $\mathrm{pH}$ dependence results from the thermodynamic coupling of the side chain ionization constants and the unfolding energy [65].

Increasing phosphorylated content led to a more intense release of piroxicam upon contact with subtilisin. As a weakly ampholytic compound, piroxicam is negatively charged at $\mathrm{pH}$ values $>5.40$ [66]. When a less hydrophobic compartment is formed and a negative charge predominate within the fibroin-based materials, piroxicam release is favored even without protease involvement. Additionally, when looser structure films are formed, the drug is able to come out more easily. A similar finding was reported, stating that the higher the crystalline content of the silk, the slower the release of the encapsulated substance [67]. Yet, the elaborated materials are dense enough to point on sub-diffusion of piroxicam as its release mechanism. Since a non-Fickian behavior was observed and the diffusion was anomaly decreased, it is reasonable that the polymers were only slightly affected by degradation. This factor is also responsible for the incomplete drug release, as it created lag phase [68], seen from the $11^{\text {th }}$ hour of incubation.

The demonstration that phospho-silk fibroin film lixiviates are not cytotoxic but do not directly promote cell proliferation is particularly interesting. The presence of very possibly newly divided cells (indicated by arrows in Fig. 5) on all the films supports this notion. Indeed, morphologically it's evident that those newly divided cells are duplets, found separately on film surface, without neighbors. These results are also in line with the previously obtained data for a L929 cell line cultivated on modified polyurethane films [69]. The determination of the film's non-cytotoxicity is a necessary step for a biomaterial to be considered biocompatible [70, 71].

To conclude, this work reports on a method for adjusting structural and biochemical features of reconstituted silk fibroin biomaterials for different uses. These modified materials can be loaded with bioactive compounds, seem to be cyto-compatible but do not actively promote cell growth or attachment. This creates the possibility of modulating drug delivery but also biological interactions. Examples of applications may be to provide support for highly differentiated cells or preventing the attachment and activation of macrophages on implant surfaces [72, 73]. Phosphorylation of silk fibroin proteins may be used to further functionalize and extend the possibilities of usage of silk for other applications.

The authors would like to acknowledge the support granted to the authors by European NOVO Project, contract no. FP7-HEALTH 2011-two-stage 278402.

The authors declare no financial or commercial conflict of interest.

\section{References}

[1] Kaplan, D., Farmer, W. A. B,Viney, C., Silk polymers: Material science and biotechnology. Polym. Adv. Technol. 1995, 6, 717.

[2] Tanaka, K., Kajiyama, N., Ishikura, K., Waga, S. et al., Determination of the site of disulfide linkage between heavy and light chains of silk fibroin produced by Bombyx mori. Biochim Biophys Acta 1999, 1432, 92-03. 
[3] Zhou, C. Z., Confalonieri, F., Jacquet, M., Perasso, R. et al., Silk fibroin: Structural implications of a remarkable amino acid sequence. Proteins 2001, 44, 119-122.

[4] Numata, K., Yamazaki, S., Naga, N., Biocompatible and biodegradable dual-drug release system based on silk hydrogel containing silk nanoparticles. Biomacromolecules 2012, 13, 1383-1389.

[5] Vepari, C., Kaplan, D. L., Silk as a Biomaterial. Prog. Polym. Sci. 2007, 32, 991-1007.

[6] Lawrence, B. D., Processing of Bombyx mori silk for biomedical applications, in: Kundu, S. (Ed.), Silk Biomaterials for Tissue Engineering and Regenerative Medicine, Elsevier, 2014, pp. 84-93.

[7] Numata, K., Kaplan, D. L., Silk-based delivery systems of bioactive molecules. Adv. Drug Delivery Rev. 2010, 62, 1497-508.

[8] Liu, X., Zhang, C., Xu, W., Liu, H., Ouyang, C., Blend films of silk fibroin and water-insoluble polyurethane prepared from an ionic liquid. Mater. Lett. 2011, 65, 2489-2491.

[9] Shang, S., Zhu, L., Fan, J., Intermolecular interactions between natural polysaccharides and silk fibroin protein. Carbohydr. Polym. 2013, 93, 561-573

[10] Wang, X., Kaplan, D. L., Functionalization of silk fibroin with NeutrAvidin and biotin. Macromol. Biosci. 2011, 11, 100-110.

[11] Karageorgiou, V., Meinel, L., Hofmann, S., Malhotra, A. et al., Bone morphogenetic protein-2 decorated silk fibroin films induce osteogenic differentiation of human bone marrow stromal cells. J. Biomed. Mater. Res. Part A 2004, 71, 528-537.

[12] Li, M., Ogiso, M., Minoura, N., Enzymatic degradation behavior of porous silk fibroin sheets. Biomaterials 2003, 24, 357-365.

[13] Freddi, G., Anghileri, A., Sampaio, S., Buchert, J. et al., Tyrosinasecatalyzed modification of Bombyx mori silk fibroin: Grafting of chitosan under heterogeneous reaction conditions. J. Biotechnol. 2006, $125,281-294$.

[14] Winkler, S., Kaplan, D. L., Molecular biology of spider silk. J. Biotechnol. 2000, 74, 85-93.

[15] Mandell, D. J., Chorny, I., Groban, E. S., Wong, S. E. et al., Strengths of hydrogen bonds involving phosphorylated amino acid side chains. J. Am. Chem. Soc. 2007, 129, 820-827.

[16] Westheimer, F. H., Why nature chose phosphates. Science 1987, 235, 1173-1178.

[17] Winkler, S., Wilson, D., Kaplan, D. L., Controlling beta-sheet assembly in genetically engineered silk by enzymatic Phosphorylation/ Dephosphorylation. Biochemistry 2000, 39, 14002.

[18] Adams, J. A., Kinetic and catalytic mechanisms of protein kinases. Chem. Rev. 2001, 101, 2271-2290

[19] Bramson, H. N., Kaiser, E. T., Mildvan, A. S., Mechanistic studies of cAMP-dependent protein kinase action. CRC Cr. Rev. Bioch. Mol. 1984, 15, 93-124.

[20] Tabata, Y., Biomaterials Design of Culture Substrates for Cell Research. Inflamm. Regen. 2011, 31, 137-145.

[21] Mieszawska, A. J., Kaplan, D. L., Smart biomaterials - regulating cell behavior through signaling molecules. BMC biology 2010, 8, 59.

[22] Dawson, E., Mapili, G., Erickson, K., Taqvi, S., Roy, K., Biomaterials for stem cell differentiation. Adv. Drug Deliv. Rev. 2008, 60, 215-228.

[23] Khorasani, M. T., Mirzadeh, H., BHK cells behaviour on laser treated polydimethylsiloxane surface. Colloids Surf. B. Biointerfaces 2004 , 35, 67-71.

[24] Hu, X., Wang, X., Rnjak, J., Weiss, A. S., Kaplan, D. L., Biomaterials derived from silk-tropoelastin protein systems. Biomaterials 2010, 31, 8121-8131

[25] Xue, Y., Ren, J., Gao, X., Jin, C. et al., GPS 2.0, a tool to predict kinasespecific phosphorylation sites in hierarchy. Mol. Cell Proteomics 2008, 7, 1598-1608.

[26] Huang, H. D., Lee, T. Y., Tzeng, S. W., Horng, J. T., KinasePhos: a web tool for identifying protein kinase-specific phosphorylation sites. Nucleic Acids Res. 2005, 33, W226-229
[27] Xue, Y., Li, A., Wang, L., Feng, H., Yao, X., PPSP: Prediction of PK-specific phosphorylation site with Bayesian decision theory. BMC bioinformatics 2006, 7, 163

[28] Kreegipuu, A., Blom, N., Brunak, S., Jarv, J., Statistical analysis of protein kinase specificity determinants. FEBS lett. 1998, 430, 45-50.

[29] Samuel, D., Silver, B. L., Elimination reactions and hydrolysis of serine phosphate. J. Chem. SoC. 1963, 289-296.

[30] Geladopoulos, T. P., Sotiroudis, T. G., Evangelopoulos, A. E., A malachite green colorimetric assay for protein phosphatase activity. Anal. Biochem. 1991, 192, 112-116.

[31] O'Toole, M., Lau, K. T., Shepherd, R., Slater, C., Diamond, D., Determination of phosphate using a highly sensitive paired emitterdetector diode photometric flow detector. Anal. Chim Acta 2007, 597, 290-294

[32] D'Angelo, E., Crutchfield, J., Vandiviere, M., Rapid, sensitive, microscale determination of phosphate in water and soil. J Environ. Qual. 2001, 30, 2206-2209.

[33] Kong, J., Yu, S., Fourier transform infrared spectroscopic analysis of protein secondary structures. Acta Biochim Biophys Sin (Shanghai) 2007, 39, 549-559.

[34] Silva, R., Ferreira, H., Carvalho, A. C., Gomes, A. C., Cavaco-Paulo, A., Protein microspheres as suitable devices for piroxicam release. Colloids Surf. B. Biointerfaces 2012, 92, 277-285.

[35] Philip L. Ritger, N. A. P., A simple equation for description of solute release I. Fickian and non-fickian release from non-swellable devices in the form of slabs, spheres, cylinders or discs. J. Controlled Release 1986, 5, 23-26

[36] Higuchi, T., Mechanism of sustained-action medication. Theoretical analysis of rate of release of solid drugs dispersed in solid matrices. J. Pharm. Sci. 1963, 52, 1145-1149.

[37] Poskus, L. T., Lima, R. S., Lima, I. R., Guimarães, J. A. et al., Cytotoxicity of current adhesive systems: in vitro testing on cell culture of L929 and balb/c 3T3 fibroblasts. Rev. odonto. cienc. 2009, 24, 129 134.

[38] Ann H. Cory, J. G. C., 5-(3-carboxymethoxyphenyl)-2-(4,5-dimethylthiazolyl)-3-(4-sulfophenyl)tetrazolium, inner salt (MTS) and related analogs of 3-(4,5-dimethylthiazolyl)-2,5-diphenyltetrazolium bromide (MTT) reducing to purple water-soluble formazans As cell-viability indicators. Bioorg. Med. Chem. Lett. 1991, 1, 611-614.

[39] Skehan, P., Storeng, R., Scudiero, D., Monks, A. et al., New colorimetric cytotoxicity assay for anticancer-drug screening. J. Natl. Cancer Inst. 1990, 82, 1107-1112.

[40] Motomizu, S., Li, Z. H., Trace and ultratrace analysis methods for the determination of phosphorus by flow-injection techniques. Talanta 2005, 66, 332-340.

[41] Tsukada M, G. Y., Nagura M, Minoura N, Kasai N, Freddi G, Structural changes of silk fibroin membranes induced by immersion in methanol aqueous solutions. J. Polym. Sci., Part B: Polym. Phys. 1994, 32, 961-968.

[42] N. V. Bhat, S. M. A., Investigation of the structure of silk film regenerated with lithium thiocyanate solution. J. Polym. Sci., Part A. Polym. Chem. 1983, 21, 1273-1280.

[43] Mo, C., Wu, P., Chen, X., Shao, Z., Near-infrared characterization on the secondary structure of regenerated Bombyx mori silk fibroin. Appl. Spectrosc. 2006, 60, 1438-1441.

[44] Silva, R. A., Yasui, S. C., Kubelka, J., Formaggio, F. et al., Discriminating 3(10)- from alpha-helices: vibrational and electronic CD and IR absorption study of related Aib-containing oligopeptides. Biopolymers 2002, 65, 229-243.

[45] Ha, S. W., Tonelli, A. E., Hudson, S. M., Structural studies of Bombyx mori silk fibroin during regeneration from solutions and wet fiber spinning. Biomacromolecules 2005, 6, 1722-1731.

[46] Fuller, A. A., Du, D., Liu, F., Davoren, J. E. et al., Evaluating beta-turn mimics as beta-sheet folding nucleators. Proc. Natl. Acad. Sci. U S A 2009, 106, 11067-11072. 
[47] Motta, A., Fambri, L., Migliaresi, C., Regenerated Silk Fibroin Films: Thermal and Dynamic Mechanical Analysis. Macromol. Chem. Phys. 2002, 203, 1658-1665.

[48] Budhavaram, N. K., Miller, J. A., Shen, Y., Barone, J. R., Protein substitution affects glass transition temperature and thermal stability. J. Agric. Food Chem. 2010, 58, 9549-9555.

[49] Bruylants, G., Wouters, J., Michaux, C., Differential scanning calorimetry in life science: thermodynamics, stability, molecular recognition and application in drug design. Curr. Med. Chem. 2005, 12, 2011-2020

[50] Magoshi, J., Magoshi, Y., Nakamura, S., Kasai, N., Kakudo, M., Physical properties and structure of silk. V. Thermal behavior of silk fibroin in the random-coil conformation. J. Polym. Sci. Polym. Phys. Ed. 1977, 15, 1675-1683.

[51] Magoshi, J., Nakamura, S., Studies on physical properties and structure of silk. Glass transition and crystallization of silk fibroin. J. Appl. Polym. Sci. 1975, 19, 1013-1015.

[52] Modhave, D. T., Handa, T., Shah, R. P., Singh, S., Successful characterization of degradation products of drugs using LC-MS tools: Application to piroxicam and meloxicam. Anal. Methods 2011, 3, 2864-2872.

[53] Costa, P., Sousa Lobo, J. M., Modeling and comparison of dissolution profiles. Eur. J. Pharm. Sci. 2001, 13, 123-133.

[54] Kamlesh K, C. S., Patit K, In-vitro Release of Metformin Hydrochloride from Films of Chitosan-Methylcellulose Blends. Asian J. Chem. 2009, 21, 148-152.

[55] Mann H.B., W. D. R., On a Test of Whether one of Two Random Variables is Stochastically Larger than the Other. Ann. Math. Stat. 1947, 18, 50-60.

[56] Liu, T. L., Miao, J. C., Sheng, W. H., Xie, Y. F. et al., Cytocompatibility of regenerated silk fibroin film: a medical biomaterial applicable to wound healing. J. Zhejiang Univ. SCi. B 2010, 11, 10-16.

[57] Mai-ngam, K., Boonkitpattarakul, K., Jaipaew, J., Mai-ngam, B., Evaluation of the properties of silk fibroin films from the non-mulberry silkworm Samia cynthia ricini for biomaterial design. J. Biomater. Sci. Polym. Ed. 2011, 22, 2001-2022.

[58] Wang, Y. W., Wu, Q., Chen, G. Q., Reduced mouse fibroblast cell growth by increased hydrophilicity of microbial polyhydroxyalkanoates via hyaluronan coating. Biomaterials 2003, 24, 4621-4629.

[59] Kiremitçi, M., Serbetçi, A. I., Çolak, R., E., P., Cell attachment to PU and PHEMA based biomaterials: Relation to structural properties. Clin. Mater. 1991, 8, 9-16.
[60] Polyansky A.A Z. B., Protein Electrostatic Properties Predefining the Level of Surface Hydrophobicity Change upon Phosphorylation. J. Phys. Chem. Lett. 2012, 3, 973-976.

[61] Ganguly, D., Chen, J., Atomistic details of the disordered states of KID and pKID. Implications in coupled binding and folding. J. Am. Chem. Soc. 2009, 131, 5214-5223.

[62] Potoyan, D. A., Papoian, G. A., Energy landscape analyses of disordered histone tails reveal special organization of their conformational dynamics. J. Am. Chem. Soc. 2011, 133, 7405-7415.

[63] Smiechowski, M., Theoretical pKa prediction of O-phosphoserine in aqueous solution. Chem. Phys. Lett. 2011, 514, 123-129.

[64] Wong Po Foo, C., Hensman, E. B. J.,Knight, D. P., Lewis, R. V., Kaplan, D. L., Role of $\mathrm{pH}$ and charge on silk protein assembly in insects and spiders. Applied Physics A 2006, 82, 223-233.

[65] Yang, A. S., Honig, B., On the $\mathrm{pH}$ dependence of protein stability. J. Mol. Biol. 1993, 231, 459-474.

[66] Takács-Novák1, K. J. K., Podányi, B., Noszál, B., Tsai, R-S. et al, Microscopic Protonation/Deprotonation Equilibria of the AntiInflammatory Agent Piroxicam. Helvetica Chimica Acta 1995, 78, 553-562.

[67] Hofmann, S., Foo, C. T., Rossetti, F., Textor, M. et al., Silk fibroin as an organic polymer for controlled drug delivery. J. Control Release 2006, 111, 219-227.

[68] Rothstein, S. N., Little, S. R., A Òtool boxÓ for rational design of degradable controlled release formulations. J. Mater. Chem 2010, 21, 29-39.

[69] Khorasani, M. T., Moemenbellah, S., Mirzadeh, H., Sadatnia, B., Effect of surface charge and hydrophobicity of polyurethanes and silicone rubbers on L929 cells response. Colloids Surf. B Biointerfaces 2006, 51, 112-119.

[70] Hezi-Yamit, A., Sullivan, C., Wong, J., David, L. et al., Impact of polymer hydrophilicity on biocompatibility: implication for DES polymer design. J. Biomed. Mater. Res. A 2009, 90, 133-141.

[71] Richards, D., Ivanisevic, A., Inorganic material coatings and their effect on cytotoxicity. Chem. Soc. Rev. 2012, 41, 2052-2060.

[72] Chiao, E., Kmet, M., Behr, B., Baker, J., Derivation of human embryonic stem cells in standard and chemically defined conditions. Method Cell Biol. 2008, 86, 1-14.

[73] Shen, M., Horbett, T. A., The effects of surface chemistry and adsorbed proteins on monocyte/macrophage adhesion to chemically modified polystyrene surfaces. J. Biomed. Mater. Res. 2001, 57, 336-345. 


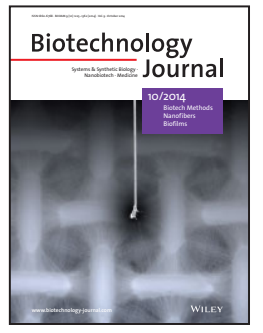

This regular issue of BT] includes the new section "Biotech Methods", and features articles on nanofibers and biofilms. The cover illustrates the technique of electrospinning, which is applied for the production of artificial filaments that can be organized to mats, which has many applications such as wound coverning or for stabilization of bioprinting/3D tissue units. It is described that silica [bio-silica] converts those mats to morphogenetically active materials. Image by Werner Mülller. See the article by Müller et al.

http://dx.doi.org/10.1002/biot.201400277

\section{Biotechnology Journal - list of articles published in the October 2014 issue.}

Editorial: "Biotech Methods" - bringing methods to the forefront of biotechnology

Judy Peng

http://dx.doi.org/10.1002/biot.201400492

Commentary

Biosilica-loaded poly( $\varepsilon$-caprolactone) nanofibers:

A step closer to bioprinted materials with tunable properties Georg M. Guebitz and Enrique Herrero Acero

http://dx.doi.org/10.1002/biot.201400383

Biotech Method

Rapid one-step purification of single-cells encapsulated in alginate microcapsules from oil to aqueous phase using a hydrophobic filter paper: Implications for single-cell experiments

Do-Hyun Lee, Miran Jang and Je-Kyun Park

http://dx.doi.org/10.1002/biot.201400319

Biotech Method

Size-selective DNA separation: Recovery spectra help determine the sodium chloride $(\mathrm{NaCl}$ ) and polyethylene glycol (PEG) concentrations required

Zhangyong He, Hong Xu, Min Xiong and Hongchen Gu http://dx.doi.org/10.1002/biot.201400234

Biotech Method

Phenylboronate chromatography selectively separates

glycoproteins through the manipulation of electrostatic, charge transfer, and cis-diol interactions

Rimenys J. Carvalho, James Woo, M. Raquel Aires-Barros,

Steven M. Cramer and Ana M. Azevedo

http://dx.doi.org/10.1002/biot.201400170

Biotech Method

Fluorescent dye ProteoStat to detect and discriminate intracellular amyloid-like aggregates in Escherichia coli

Susanna Navarro and Salvador Ventura

http://dx.doi.org/10.1002/biot.201400291

Biotech Method

Phosphorylation of silk fibroins improves the cytocompatibility

of silk fibroin derived materials: A platform for the production

of tuneable materials

Vadim Volkov, Andreia Vasconcelos, Marisa P. Sárria,

Andreia C. Gomes, Artur Cavaco-Paulo

http://dx.doi.org/10.1002/biot.201400302

Research Article

A functional high-content miRNA screen identifies miR-30 family to boost recombinant protein production in $\mathrm{CHO}$ cells Simon Fischer, Theresa Buck, Andreas Wagner, Carolin Ehrhart, Julia Giancaterino, Samuel Mang, Matthias Schad, Sven Mathias, Armaz Aschrafi, René Handrick and Kerstin Otte

http://dx.doi.org/10.1002/biot.201400306

\section{Research Articles}

Feeding strategies enhance high cell density cultivation and protein expression in milliliter scale bioreactors Georg Faust, Nils H. Janzen, Christoph Bendig, Lin Römer, Klaus Kaufmann and Dirk Weuster-Botz http://dx.doi.org/10.1002/biot.201400346

Research Article

Bioactive nanoparticles stimulate bone tissue formation in bioprinted three-dimensional scaffold and human mesenchymal stem cells

Guifang Gao, Arndt F. Schilling, Tomo Yonezawa, Jiang Wang, Guohao Dai and Xiaofeng Cui

http://dx.doi.org/10.1002/biot.201400305

Research Article

Biosilica-loaded poly(e-caprolactone) nanofibers mats provide a morphogenetically active surface scaffold for the growth and mineralization of the osteoclast-related SaOS-2 cells Werner E.G. Müller, Emad Tolba, Heinz C. Schröder, Bärbel Diehl-Seifert, Thorben Link and Xiaohong Wang http://dx.doi.org/10.1002/biot.201400277

\section{Research Article}

High-level conversion of L-lysine into 5 -aminovalerate that can be used for nylon 6,5 synthesis

Si Jae Park, Young Hoon Oh, Won Noh, Hye Young Kim, Jae Ho Shin, Eun Gyo Lee, Seungwoon Lee, Yokimiko David, Mary Grace Baylon, Bong Keun Song, Jonggeon Jegal, Sang Yup Lee and Seung Hwan Lee

http://dx.doi.org/10.1002/biot.201400156

Research Article

Synergistic effect of Aspergillus niger and Trichoderma reesei enzyme sets on the saccharification of wheat straw and sugarcane bagasse

Joost van den Brink, Gabriela Piccolo Maitan-Alfenas, Gen Zou, Chengshu Wang, Zhihua Zhou, Valéria Monteze Guimarães and Ronald P. de Vries

http://dx.doi.org/10.1002/biot.201400317

Research Article

Solid support membrane-aerated catalytic biofilm reactor for the continuous synthesis of (S)-styrene oxide at gram scale Babu Halan, Thomas Letzel, Andreas Schmid and Katja Buehler

http://dx.doi.org/10.1002/biot.201400341

Research Article

Metabolic modeling of spatial heterogeneity of biofilms in microbial fuel cells reveals substrate limitations in electrical current generation

Nadeera Jayasinghe, Ashley Franks, Kelly P. Nevin

and Radhakrishnan Mahadevan

http://dx.doi.org/10.1002/biot.201400068 